\title{
Participación en investigación durante la formación en Traumatología y Ortopedia en Chile ¿Con qué frecuencia publican los residentes?
}

\section{Research Involvement during Orthopedic Surgery Training in Chile. How Frequently Do Residents Publish?}

\author{
Jorge Cabrolier ${ }^{1,2}$ Carlos Gomez ${ }^{1,2}$ Rodrigo Guiloff ${ }^{1,2}$ Martin Leyton ${ }^{2} \quad$ David Figueroa $^{1}$
}

${ }^{1}$ Departamento de Traumatología y Ortopedia, Clínica Alemana de Santiago, Santiago, Chile

2 Facultad de Medicina, Universidad del Desarrollo, Santiago, Chile Rev Chil Ortop Traumatol 2021;62(2):e99-e103.
Address for correspondence Jorge Cabrolier, MD, Departamento de Traumatología y Ortopedia, Clínica Alemana de Santiago, Avenida Vitacura 5.951, Vitacura, Región Metropolitana, Santiago, Chile (e-mail: jcabrolierj@gmail.com).

\section{Resumen \\ Objetivo Determinar el promedio de artículos indexados publicados por residentes de traumatología en Chile y, en segundo lugar, establecer si el tiempo protegido para investigación se asociaba con un mayor número de publicaciones. \\ Métodos Se desarrolló un estudio descriptivo transversal. Se realizó una búsqueda en la base de datos de PubMed para identificar publicaciones escritas por residentes egresados de programas chilenos entre 2012 y 2016 . Se incluyeron artículos con fecha de publicación entre 6 meses después del inicio de su residencia y 12 meses después de la graduación. Se excluyeron las cartas al editor, los artículos no relacionados con la cirugía ortopédica, y los proyectos de investigación con una afiliación institucional distinta a sus programas de formación. Cada currículo de los programas de residencia fue revisado para que se identificara la mención de tiempo protegido para investigación. \\ Resultados Se incluyó a un total de 272 residentes de 10 programas, y 72 artículos cumplieron los criterios de selección. La tasa de publicación promedio fue de 0,26 $\pm 1,13$ artículos (rango: 0 a 13 artículos) por residente durante su formación. En total, 2 programas tuvieron una tasa de publicación promedio superior a 1, y un 89,4\% de los residentes chilenos no publicó ningún artículo durante su formación. \\ Palabras clave \\ - residente \\ - investigación \\ - ortopedia \\ Ninguno de los programas especificó tiempo protegido para la investigación. \\ Conclusiones A nuestro entender, este es el primer estudio que describe el estado actual de la participación en la investigación entre los residentes de traumatología chilenos. El número promedio de publicaciones indexadas en PubMed por residente}

recibido

29 de agosto de 2020

aceptado

02 de junio de 2021
DOI https://doi.org/

10.1055/s-0041-1734012. ISSN $0716-4548$. (c) 2021. Sociedad Chilena de Ortopedia y Traumatologia. All rights reserved.

This is an open access article published by Thieme under the terms of the Creative Commons Attribution-NonDerivative-NonCommercial-License, permitting copying and reproduction so long as the original work is given appropriate credit. Contents may not be used for commercial purposes, or adapted, remixed, transformed or built upon. (https://creativecommons.org/ licenses/by-nc-nd/4.0/)

Thieme Revinter Publicações Ltda., Rua do Matoso 170, Rio de Janeiro, RJ, CEP 20270-135, Brazil 
durante la formación en Chile fue de $0.26 \pm 1.13$ artículos. Sólo el $10 \%$ de los residentes publica un artículo durante su residencia. No se pudo establecer una correlación entre el tiempo protegido para la investigación y el número de publicaciones.

Abstract

Keywords

- resident

- research

- orthopedics
Objective To determine the average number of indexed articles published per orthopedic resident in Chile, and, secondarily, to establish if protected research time for residents was associated with a higher number of publications.

Methods A transverse descriptive study was developed. A search for publications authored by orthopedic residents who graduated from Chilean programs between 2012 and 2016 was performed on the PubMed database. Articles with a publication date between 6 months after the beginning of the residency and 12 months postgraduation were included. Letters to the editor, articles not related to orthopedic surgery, and research projects with an institutional affiliation other than their training programs were excluded. Each residency curriculum was reviewed for protected time for research.

Results A total of 272 residents from 10 programs were included, and 72 articles fulfilled the selection criteria. The average rate of publication was of $0.26 \pm 1.13$ articles (range: 0 to 13 articles) per resident during training. In total, 2 programs had an average publication rate higher than 1 , and $89.4 \%$ of the Chilean residents did not publish an article during their training. None of the programs specified protected time for research.

Conclusions To our understanding, the present is the first study to describe the current state of research involvement among Chilean orthopedic residents. The average number of publications indexed on PubMed per orthopedic resident during training was of $0.26 \pm 1.13$ articles. Only $10 \%$ of the residents publish an article during their residency. No correlation between protected time for research and the number of publications could be established.

\section{Introducción}

La participación en la investigación se ha convertido en un aspecto fundamental durante la formación de residencia de los cirujanos ortopédicos, ya que ha demostrado desarrollar habilidades de pensamiento crítico que son necesarias para la práctica clínica. ${ }^{1-4}$ El Accreditation Council for Graduate Medical Education (Consejo de Acreditación para la Educación Médica de Graduados, ACGME, en inglés) y el Royal College of Physicians and Surgeons of Canada (Colégio Real de Médicos y Cirujanos de Canadá, CanMEDS, en inglés) abogan por programas de formación para fomentar la participación en la investigación. ${ }^{5,6}$ En consecuencia, se ha incrementado el interés por las variables que inciden en la tasa de publicación de los residentes de ortopedia. ${ }^{2,3,7}$ El tiempo protegido para investigación parece jugar un papel significativo en la tasa de publicación, que es significativamente diferente entre los residentes de diferentes programas. ${ }^{5,7}$ El mismo interés por las publicaciones de residentes se ha reportado en los países de la América Latina. ${ }^{8,9}$ Sin embargo, un estudio reciente ${ }^{10}$ mostró que solo el $1 \%$ de la literatura ortopédica publicada entre 1988 y 2013 se originó en esta región. A partir de esta evidencia, surgen diferentes preguntas: ¿los residentes de ortopedia de la América Latina participan activamente en la investigación? ¿Cuántos artículos publican? ¿Existe una disparidad con los países de la América del Norte? Por lo que sabemos, no hay suficientes datos publicados para responder a estas preguntas. El objetivo principal del presente estudio fue determinar el promedio de artículos indexados publicados en Chile por ortopedistas residentes y, en segundo lugar, establecer si el tiempo de investigación protegido para los residentes se asoció con un mayor número de publicaciones.

\section{Métodos}

Se desarrolló un estudio descriptivo transversal. La Sociedad Chilena de Cirugía Ortopédica (SCHOT) entregó una lista de todos los programas de residencia en ortopedia en Chile. Se revisó el plan de estudios de cada institución, y se registró cualquier mención de tiempo protegido para la investigación. Se registraron los nombres completos de los residentes que se graduaron entre 2012 y 2016, y, si un programa no tuvo graduados en este período, se excluyó del análisis. Por último, se contactó directamente con cada programa para confirmar la información.

Criterios de búsqueda: se incluyeron artículos indexados en PubMed de autoría o coautoría de los residentes de ortopedia con una fecha de publicación entre 6 meses 
después del inicio de su formación y 12 meses después de la graduación. Fueron excluídos las cartas al editor, los artículos no relacionados con la cirugía ortopédica, y aquellos con una afiliación institucional distinta a su programa de formación. Se buscó el nombre y apellido de cada residente en la base de datos electrónica. Para evitar datos incorretos debido a la posibilidad de que varios investigadores tengan los mismos nombres, también se verificaron los nombres y las afiliaciones institucionales de los coautores.

Se registró el número de artículos publicados por cada residente para determinar la tasa de publicación por residente y por programa. Enseguida, los artículos fueron analisados según el factor de impacto de cada revista (en el momento de la revisión) y el nivel de evidencia de cada uno. Los ensayos clínicos se clasificaron de acuerdo con los niveles de evidencia del Oxford Center for Evidence-Based Medicine. ${ }^{11}$

Dos investigadores independientes realizaron esa búsqueda electrónica en agosto de 2017. Todos los datos se almacenaron utilizando Google Sheet (Alphabet), y el análisis de datos se realizó utilizando el software Stata (StataCorp, LLC, College Station, TX, EEUU), versión 12.0.

\section{Fuente de Financiación}

No se recibió financiación de ninguna fuente para esta investigación.

\section{Resultados}

Se identificaron doce programas de residencia en cirugía ortopédica en Chile, todos con una duración de formación de tres años. Dos fueron excluidos porque ningún residente se había graduado de ellos en el momento del análisis. En total, se incluyeron 272 residentes, con un promedio de 27,2 $\pm 22,9$ (rango: 7 a 85) residentes por programa. Los criterios de inclusión fueron cumplidos por 72 estudios (se excluyeron 2 artículos por ser cartas al editor). Los estudios fueron escritos por $29(10,6 \%)$ de los 272 residentes. La tasa de publicación fue de $0,26 \pm 1,13$ (rango: 0 a 13) artículos por residente durante la formación. En total, destacaron 2 programas, con una media de más de 1 artículo publicado por residente $(2,6 \mathrm{y}$ 1,3 artículos). La mitad de los programas contaba con al menos un residente que había publicado un artículo durante su residencia (-Fig. 1). Entre los artículos publicados, hubo 42 ensayos clínicos, 12 estudios experimentales, y 18 informes, incluidas guías de técnicas quirúrgicas y la validación de medidas de resultados clínicos y sistemas de clasificación de enfermedades. El nivel de evidencia de cada ensayo clínico se muestra en la - Tabla 1, y la mayoría corresponde a estudios de nivel IV o V (78,5\%). Las revistas en las que más publicaron los residentes chilenos y sus respectivos factores de impacto se resumen en la - Tabla 2. Ninguno de los diez programas de capacitación mencionó el tiempo protegido para la investigación; por lo tanto, no se pudo realizar ningún análisis para establecer si la existencia de tiempo protegido para la investigación se correlacionaba con el número de publicaciones.

\section{Discusión}

Existe un creciente interés por la investigación en los países de la América Latina, ${ }^{8,9}$ pero el número de publicaciones sobre ortopedia indexadas en esta región aún es escaso en comparación con otras. ${ }^{10}$ Incentivar a los residentes a

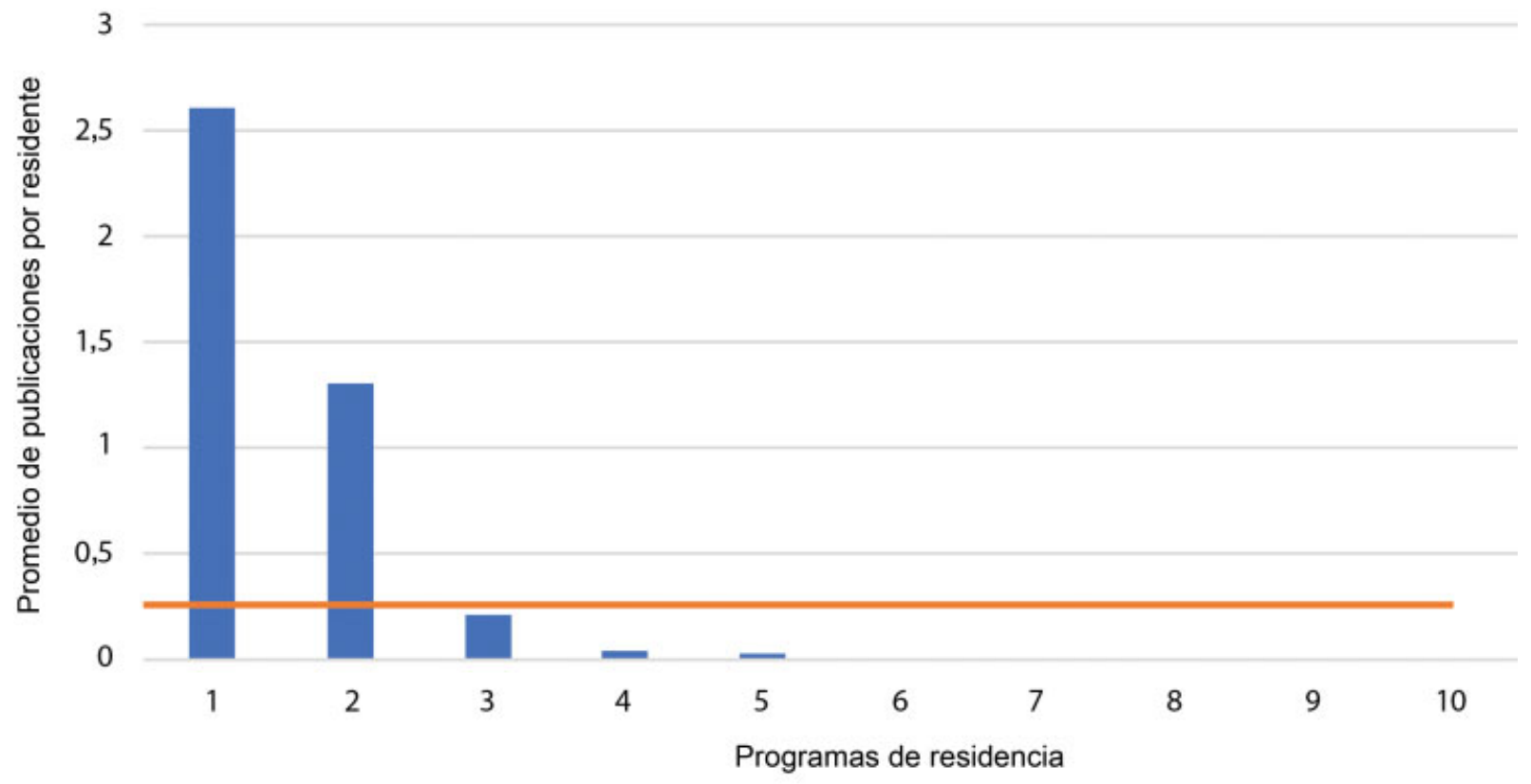

Tasa de publicación del programa _ Tasa media de publicaciones

Fig. 1 Número medio de artículos publicados por residente por programa. La línea naranja indica la tasa de publicación promedio de todos los residentes. 
102 Participación en investigación durante la formación en Traumatología y Ortopedia en Chile Cabrolier et al.

Tabla 1 Proporción de artículos de ensayos clínicos incluidos según su nivel de evidencia (Oxford Centre for Evidence-Based Medicine Levels of Evidence)

\begin{tabular}{|l|l|}
\hline Nivel de evidencia & Número de publicaciones \\
\hline I & 0 \\
\hline II & 4 \\
\hline III & 5 \\
\hline IV & 29 \\
\hline V & 4 \\
\hline
\end{tabular}

Tabla 2 Las diez revistas más frecuentes con publicaciones de residentes

\begin{tabular}{|l|l|l|}
\hline Revista & $\begin{array}{l}\text { Scimago Journal \& } \\
\text { Country Rank }\end{array}$ & $\begin{array}{l}\text { Número de } \\
\text { publicaciones }\end{array}$ \\
\hline Spine & 1,736 & 10 \\
\hline $\begin{array}{l}\text { European Spine } \\
\text { Journal }\end{array}$ & 1,535 & 8 \\
\hline $\begin{array}{l}\text { Revista Española de } \\
\text { Cirugía Ortopédica }\end{array}$ & 0,28 & 8 \\
\hline Knee & 1,244 & 7 \\
\hline $\begin{array}{l}\text { International } \\
\text { Orthopaedics }\end{array}$ & 1,502 & 5 \\
\hline Arthroscopy & 1,459 & 5 \\
\hline $\begin{array}{l}\text { Foot and Ankle } \\
\text { International }\end{array}$ & 1,626 & 4 \\
\hline Injury & 0,99 & 4 \\
\hline $\begin{array}{l}\text { Revista Médica de } \\
\text { Chile }\end{array}$ & 0,26 & 4 \\
\hline $\begin{array}{l}\text { American Journal of } \\
\text { Sports Medicine }\end{array}$ & 3,949 & 3 \\
\hline
\end{tabular}

realizar investigación científica podría ser de gran interés para estos países, ya que se ha demostrado que los residentes que publican durante sus programas de formación tienden a seguir trabajando en proyectos de investigación a lo largo de su carrera. ${ }^{4,6,12,13}$ Sin embargo, no sabemos qué proporción de residentes realmente logra publicar un artículo con éxito. A nuestro entender, no hay informes que describan la participación de los residentes de cirugía ortopédica de la América Latina en investigaciones.

Los resultados del presente estudio mostraron que un residente chileno de cirugía ortopédica publica en promedio $0,26 \pm 1,13$ artículos en un período de 3 años. Un estudio reciente $^{7}$ de los Estados Unidos, que incluyó a 1.690 residentes de 125 programas de ortopedia, mostró una tasa de publicación de 1,2 $\pm 2,4$ artículos por residente en un período de 5 años. Entre los residentes de ortopedia canadienses, la tasa de publicación durante 1 año de formación fue de $0,42 \pm 0,9$, que también es superior a la tasa chilena. ${ }^{5}$ Múltiples factores podrían explicar esta diferencia, como la duración total de la formación (cinco años versus tres años), el idioma, un programa de investigación dedicado, ${ }^{14,15}$ el compromiso de tutoría del profesorado, 4,14,16,17 el acceso a un editor médico de investigación, la provisión de becas, ${ }^{14}$ la posibilidad de ser el autor principal, ${ }^{18}$ la posibilidad de un año de investigación electivo, ${ }^{19}$ restricciones de horas de trabajo, ${ }^{5,20,21}$ y tiempo protegido para la investigación. 5,7,14

Podría ser de interés aplicar algunos de estos factores en los programas de residencia local, ya que su reestructuración ha dado lugar a un aumento significativo de subvenciones y publicaciones en otras regiones como la América del Norte. $^{14,15,22}$ Robbins et al., ${ }^{14}$ por ejemplo, mostraron un aumento en la tasa de publicación de 1,3 a 9,3 artículos por residente durante la formación ortopédica después de ajustes en el plan de estudios de investigación.

En cuanto al tiempo protegido para la investigación, el estudio de Williams et al. ${ }^{7}$ mostró que los residentes estadounidenses de cirugía ortopédica que tenían tiempo designado para la investigación publicaron significativamente más que los que no lo tenían. Lo mismo ha sido demostrado por Chan et al. ${ }^{5}$ con respecto a los residentes de ortopedia canadienses, con una media de tiempo protegido de $5 \pm 7,3$ meses. En su estudio, ${ }^{5}$ también mostraron que el $41 \%$ de los residentes encuestados respondieron que el tiempo era el mayor obstáculo para la investigación. En el presente análisis, ninguno de los programas chilenos informó tiempo protegido para la investigación.

Es importante resaltar que existe una amplia gama de artículos publicados entre residentes chilenos ( 0 a 13 publicaciones). Al comparar los diferentes programas de residencia, identificamos que dos de ellos concentraban a los residentes que más publicaban. Estos fueron los únicos con una tasa de publicación de más de un artículo por residente, alcanzando el promedio norteamericano. ${ }^{7}$ En los otros ocho programas, la tasa de publicación fue cercana a cero. Las posibles explicaciones para estas diferencias incluyen las variables antes mencionadas, pero también creemos que la experiencia de investigación del estudiante también es un factor que podría impactar las tasas de publicación, una vez que algunos programas pueden tener una mayor proporción de residentes que ya tienen una experiencia inicial y interés en la investigación.

El análisis de los artículos publicados por los residentes chilenos mostró que muchos fueron publicados en revistas de ortopedia de alto impacto (-Tabla 1). Sin embargo, esto no garantiza necesariamente la calidad del artículo. ${ }^{15}$ Además, la mayoría de los ensayos clínicos incluidos en el presente estudio $(78,5 \%)$ tenían un nivel de evidencia de IV o V (-Tabla 2).

El presente estudio tiene limitaciones que pueden haber dado lugar a sesgos de selección. Primero, la búsqueda en la base de datos se realizó exclusivamente en PubMed; por lo tanto, se excluyeron las publicaciones que no estaban indexadas en PubMed. Esta base de datos se utilizó porque es el principal motor de búsqueda de evidencia científica, ${ }^{23}$ incluye todas las revistas principales, ${ }^{24}$ y es consistente con los métodos de la literatura actual. ${ }^{5,7}$ En segundo lugar, el límite de tiempo establecido para los criterios de inclusión de los artículos publicados (6 meses después del inicio de la residencia hasta 12 meses después de la graduación) se 
definió arbitrariamente, de manera que se asegurara sólo la inclusión de artículos que fueron desarrollados por los residentes durante su formación. Estos criterios pueden haber causado que la tasa de publicación esté subestimada o sobrevalorada. Al igual que con varios estudios descriptivos, la generalización de nuestros resultados también es limitada, porque algunas comparaciones se realizaron utilizando la literatura publicada. Para un análisis más preciso, las investigaciones futuras podrían enfocarse en la realización de estudios comparativos transversales entre residentes de ortopedia latinoamericanos y norteamericanos.

Con base en nuestro conocimiento, el presente es el primer estudio que reporta el estado actual de la participación en la investigación y el número de publicaciones de ortopedistas residentes en un país de la América Latina. Los próximos estudios en Chile deben enfocarse en identificar las diferencias entre los programas de residencia en ortopedia, para reconocer las variables clave que llevan a un grupo de estudiantes de un mismo país a destacarse entre sus pares. También sería valioso evaluar la opinión del residente sobre los factores que influyen en su éxito en el desarrollo y en la publicación de la investigación.

\section{Conclusiones}

El presente estudio determinó que el número promedio de publicaciones indexadas en PubMed por residentes de ortopedia en Chile es de 0,26 $\pm 1,13$ artículos, menor al de los ortopedistas residentes norteamericanos. Solo el 10\% de ellos publica un artículo durante su residencia. Ninguno de los programas chilenos especificó un tiempo protegido para la investigación; por eso, no se pudo establecer correlación entre esta variable y el número de artículos publicados por residentes.

Conflicto de interés

Los autores declaran que no hay conflicto de intereses.

\section{Referencias}

1 Atesok KI, Hurwitz SR, Egol KA, et al. Perspective: Integrating research into surgical residency education: lessons learned from orthopaedic surgery. Acad Med 2012;87(05):592-597

2 Bishop JA. CORR Insights ${ }^{\circledR}$ : A dedicated research program increases the quantity and quality of orthopaedic resident publications. Clin Orthop Relat Res 2015;473(04):1522-1523

3 Bernstein J. CORR Insights ${ }^{\circledR}$ : Does research participation make a difference in residency training? Clin Orthop Relat Res 2014;472 (01):377-378

4 Bechtold JE, Williams BR, Weinstein SL, et al. The Pursuit of Scholarship: Why We Should Care About Resident Research. J Bone Joint Surg Am 2017;99(22):e119

5 Chan RKW, Lockyer J, Hutchison C. Block to succeed: the Canadian orthopedic resident research experience. Can J Surg 2009;52(03): 187-195

6 ACGME Program Requirements for Graduate Medical Education in Orthopaedic Surgery. https://www.acgme.org/Portals/0/PFAssets/ ProgramRequirements/260_OrthopaedicSurgery_2017-07-01.pdf?ver= 2017-06-14-093103-267
7 Williams BR, Agel JA, Van Heest AE. Protected Time for Research During Orthopaedic Residency Correlates with an Increased Number of Resident Publications. J Bone Joint Surg Am 2017;99 (13): e73

8 Concha M, Miranda J C, Vergara G, Ibarra H. Intención y desarrollo de competencias en investigación clínica en programas de postítulo de médico especialista en Chile. Rev Med Chil 2012; 140(03):326-333

9 Falavigna A. Strategy to Increase Research in Latin America: Project on Education in Research by AOSpine Latin America. Global Spine J 2015;5(04):264-265

10 Urrutia J, Zamora T, Prada C. The fifty most cited Latin-American articles in the orthopaedic literature. Int Orthop 2014;38(08): 1723-1729

11 CEBM (Centre for Evidence-Based Medicine) Oxford Centre for Evidence-Based Medicine -Levels of Evidence. https://www.cebm. net/2009/06/oxford-centre-evidence-basedmedicine-levels-evidencemarch-2009/. Publicado en 2009. Acesado en 20 de Junio de 2017

12 Macknin JB, Brown A, Marcus RE. Does research participation make a difference in residency training? Clin Orthop Relat Res 2014;472(01):370-376

13 Konstantakos EK, Laughlin RT, Markert RJ, Crosby LA. Assuring the research competence of orthopedic graduates. J Surg Educ 2010; 67(03):129-134

14 Robbins L, Bostrom M, Marx R, Roberts T, Sculco TP. Restructuring the orthopedic resident research curriculum to increase scholarly activity. J Grad Med Educ 2013;5(04):646-651

15 Torres D, Gugala Z, Lindsey RW. A dedicated research program increases the quantity and quality of orthopaedic resident publications. Clin Orthop Relat Res 2015;473(04):1515-1521

16 Pellegrini VDJ Jr. Mentoring during residency education: a unique challenge for the surgeon? Clin Orthop Relat Res 2006; 449:143-148

17 Manring MM, Panzo JA, Mayerson JL. A framework for improving resident research participation and scholarly output. J Surg Educ 2014;71(01):8-13

18 Ahn J, Donegan DJ, Lawrence JTR, Halpern SD, Mehta S. The future of the orthopaedic clinician-scientist: part II: Identification of factors that may influence orthopaedic residents' intent to perform research. J Bone Joint Surg Am 2010;92(04):1041-1046

19 Segal LS, Black KP, Schwentker EP, Pellegrini VD. An elective research year in orthopaedic residency: how does one measure its outcome and define its success? Clin Orthop Relat Res 2006; 449:89-94

20 DeHaven MJ, Wilson GRO-KP, O'Connor-Kettlestrings P. Creating a research culture: what we can learn from residencies that are successful in research. Fam Med 1998;30(07):501-507

21 Levitt MA, Terregino CA, Lopez BL, Celi C. Factors affecting research directors' and residents' research experience and productivity in emergency medicine training programs. Acad Emerg Med 1999;6(04):356-359

22 Kohlwes J, O'Brien B, Stanley M, et al. Does Research Training During Residency Promote Scholarship and Influence Career Choice? A Cross-Sectional Analysis of a 10-Year Cohort of the UCSF-PRIME Internal Medicine Residency Program. Teach Learn Med 2016;28(03):314-319

23 Falagas ME, Pitsouni EI, Malietzis GA, Pappas G. Comparison of PubMed, Scopus, Web of Science, and Google Scholar: strengths and weaknesses. FASEB J 2008;22(02):338-342

24 Halladay CW, Trikalinos TA, Schmid IT, Schmid CH, Dahabreh IJ. Using data sources beyond PubMed has a modest impact on the results of systematic reviews of therapeutic interventions. J Clin Epidemiol 2015;68(09):1076-1084 